\title{
Talking PACS: Part 1 - What is PACS?
}

\author{
Otto Carl Schulze, $M B C h B$ \\ Jaco Greyling, $M B C h B, D A(S A), B S c$ Hons \\ (Pharmacology) \\ Murray Hayes, $M B C h B$ \\ Savvas Andronikou, MB BCh, FCRad, FRCR, PhD \\ Department of Radiology, Stellenbosch University, Tygerberg
}

\section{Abstract}

Medical image generating departments, typically radiology and nuclear medicine, have always been burdened by image management problems. Image management is the administration of imaging examination requests, the performance of imaging examinations, the distribution of the images and reports, the archiving of examinations and results and the retrieval of previous examinations and results. This is a very resource-intensive responsibility dependent on a long chain of human interaction that often fails.

Within both the public and private sectors this is a real problem. In the public sector increased efficiency is required to decrease the waiting lists and improve patient care. In the private sector improved productivity is required to increase the revenue generated by the same amount of human and financial resources. The solution is to embrace digital image management.

The solution does not lie within a single information system, but rather the tight integrations of four parts: digital imaging equipment, PACS (Picture Archiving and Communication System), RIS (Radiological Information System) and HIS (Hospital Information System)

This article aims to demystify some of the acronyms commonly used and explain their practical role within the classic imaging department.

Where is the $\mathrm{X}$-ray? Are you still waiting for the filing room or the scheduling of the examination, the examination to be performed or are you waiting for the report?

If any of the above questions frustrate you in your practice then you are ready for the next step in medical imaging - PACS (Picture Archiving and Communication System).

Medical image generating departments, typically radiology and nuclear medicine, have always been burdened by image management problems. Image management is the administration of imaging examination requests, the performance of imaging examinations, the distribution of the images and reports, the archiving of examinations and results and the retrieval of previous examinations and results. This is a very resource-intensive responsibility dependent on a long chain of human interaction that often fails.
This problem has grown substantially, firstly because of an increasing demand for imaging examinations, specifically specialised imaging examinations, e.g. magnetic resonance imaging (MRI) and multi-detector computed tomography (MDCT), and secondly as a result of an exponential increase in the number of images generated per examination. This necessitates a radical redesign of the image management process.

Imagine requesting an investigation using your personal computer and being able to track the request progress as it is approved, scheduled and performed as demonstrated in Fig. 1. Imagine being able to view the same investigation immediately after it has been performed at a remote site (e.g. your office, ward or surgical theatre), on your own computer again. Imagine also reading the attached report as soon as the radiologist completes it, as demonstrated in Fig. 2. Well, you only have to imagine this if you haven't installed such a system in your hospital because this is what modern PACS offers you. To understand PACS better one needs to be familiar with some of the jargon and abbreviations in use. We have attached a workflow map to help you orientate yourself with all the terms defined and explained below:

\section{PACS: Picture Archiving and Communication System ${ }^{1}$}

This is an information system responsible for the storage, distribution and viewing of digital images, consisting of:

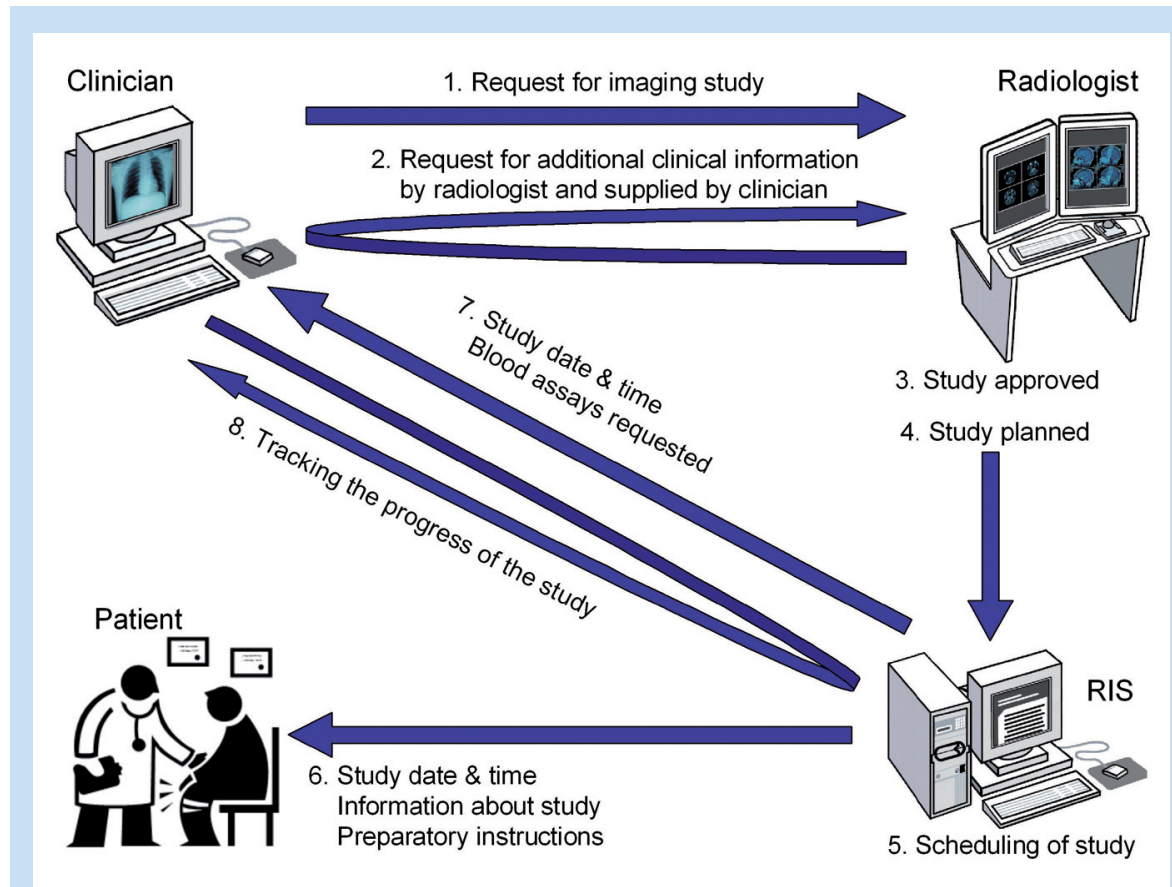

Fig. 1. This is a diagrammatic representation of the ordering of an imaging examination workflow. Step 1 shows the clinician requesting an examination. Step 2 shows the radiologist enquiring about more clinical information to plan the optimal examination. Step 3 shows the radiologist approving the request and in Step 4 he plans the examination. Step 5 shows the automatic scheduling of the examination. Step 6 shows the feedback given to the patient regarding the date and time of the examination, more information about the procedure (what it would entail, what are the risks \& what are the benefits) and also instructions for the patient regarding preparatory steps to optimise the examination (bowel preparation, blood assays). Step 7 shows the feedback given to the clinician regarding the date and time of the examination as well as additional blood assays required before the examination. Step 8 shows the ability of the clinician to track the progress of the examination at any time, e.g. received for approval, awaiting clinical info, approved, scheduled, patient arrived, performed, verified, reported and signed. 
- Hardware: imaging modalities, network, server, archive and stations

- Software that performs the following functions:

- Automatic archiving

- Viewing and image manipulation software

- Automatic distribution of examination results

\section{DICOM: Digital Imaging and Communication in Medicine ${ }^{2}$}

This is the standard of communication between different devices managing digital images, including: imaging modalities, printers, stations and servers.

This is the part of the PACS that is likened to the X-ray film format, e.g. film of the same speed and light sensitivity.

\section{Modalities}

These are the different types of imaging devices.

- Most of the images are generated digitally, e.g. MDCT \& MRI.

- Some of the images were traditionally generated as an analogue signal (you could attach your video recorder or television to it) and then changed to the DICOM format; like ultrasound, cardiac \& angio-theatre. Nowadays most of these are generated digitally.

- Lastly, there is projection radiography, e.g. plain film and mammography. To digitise these images there are three options:

- Digitiser: this is a scanner for X-ray films, converting them into a digital format.

- CR: computed radiography. In this modality there is an X-ray sensitive plate within a cassette, which captures the X-rays. Cassettes can be used in existing X-ray equipment. After being exposed, the cassette is then fed into a cassette reader, which extracts the X-ray image from the plate and erases the plate for repeat use. This is a low-cost solution.

- DR: digital radiography. In this modality there is no cassette, the patient is positioned against an X-ray sensitive panel and the X-ray image is captured directly. This is a high-cost solution with the advantage of having better image quality. It compares to a digital camera where the image is immediately stored digitally without requiring development.

\section{Computer networks ${ }^{3}$}

- Communication lines between digital devices can be established by using electrical impulses via UTP (unshielded twisted pair) cable or light pulses via optical fibre or radio waves via wireless connectivity. The Cable and optical fibre are able to achieve much higher transfer speeds, optical fibre is able to transmit over much longer distances and wireless options give you the advantage of being mobile but are lacking in transfer speed and distance.
- Communication speeds run at 10, 100 or $1000 \mathrm{Mbit} / \mathrm{s}$. Note that 100 $\mathrm{Mbit} / \mathrm{s}$ means $100 / 8 \mathrm{Mbyte} / \mathrm{s}$ ( 8 bits $=1$ byte) thus about $12.5 \mathrm{Mbytes}$ per second. The communication speed on a specific network is determined by the connection points (explained below), the communication lines between the computers and the connection points and the network cards within these computers.

- There are two types of connection points, which differ in the way they share the connection speed between the connections. The first is called a hub, which shares the maximum network speed among all the connections; thus the more people connected to the network, the slower it becomes. The second is a switch, which allows each channel to access the maximum network speed, thus regardless of how many people are connected to the network the network speed remains constant.

This is the part of the PACS that is likened to the porter carrying the request form, the $X$-rays and the report or the telephone network connecting referring doctors, radiographers, clerks and radiologists to each other.

\section{LAN: Local Area Network ${ }^{3}$}

This refers to a computer network within a limited geographic region (e.g. a room, building or campus). The defining characteristics of LANs are their much higher data rates, smaller geographic range, and that they do not require leased telecommunication lines.

\section{WAN: Wide Area Network ${ }^{3}$}

This refers to a computer network encompassing a wide/broad geographic area e.g. cities, provinces, and countries. The largest and most well known example of a WAN is the Internet. WANs are used to connect local area 


\begin{tabular}{llll}
\hline Modality & Size per image & Average number of images per examination & Total size per examination \\
\hline $\mathrm{CT}$ & $0.5 \mathrm{MB}$ & $20-3000$ & $10-1500 \mathrm{MB}$ \\
$\mathrm{MRI}$ & $0.5 \mathrm{MB}$ & $100-200$ & $50-100 \mathrm{MB}$ \\
CR/DR & $20-30 \mathrm{MB}$ & 2 & $40-60 \mathrm{MB}$ \\
Fluoroscopy & $1 \mathrm{MB}$ & $100-200$ & $100-200 \mathrm{MB}$ \\
Ultrasound (single images) & $0.7 \mathrm{MB}$ & $3-15$ & $2.1-10.5 \mathrm{MB}$ \\
Angiography & $2 \mathrm{MB}$ & $100-200$ & $200-400 \mathrm{MB}$
\end{tabular}

networks (LANs) together, so that users and computers in one location can communicate with users and computers in other locations.

\section{VPN: Virtual Private Network ${ }^{4}$}

This refers to a private communications network used by an organisation which is spread across a wide geographic area, wanting to communicate confidentially over a publicly accessible network (e.g. the Internet), thus creating a confidential WAN.

\section{Station $^{5}$}

This refers to a computer interface to gain access to the PACS. There are different station profiles with different levels of functionality.

- Diagnostic workstation: high-end workstation used by radiologists. It consists of 2 or 3 monitors, normally 2 high-resolution grey-scale screens used for viewing of most imaging and one colour screen used for advanced image manipulation (3D reconstructions) and interfacing with the RIS (Radiological Information System).

This is the part of the PACS that is likened to the array of viewing boxes and hot lamps and magnifying glasses usually found within a radiology department.

- Viewing station: lower-end viewing station used by clinicians in the wards, clinics and theatres. The quality of viewing is preserved but the manipulation capabilities are restricted. It normally consists of just one colour monitor.

This is the part of the PACS that is likened to the viewing boxes within the wards, clinics and theatres.

- Quality control station: used by radiographers to operate an imaging modality and confirm the quality of a recently performed examination. This is the part of the PACS that is likened to the viewing box in the X-ray room that the radiographers use for quality control.

\section{Server}

This is a high-end computer that controls all the functions of the PACS or RIS. The PACS server contains and manages the image database. The RIS server contains and manages the workflow database. Refer to Figs 1 and 2 for an illustration of the workflow.

This part is likened to the office of the administrative head.

\section{Archive}

This is a computer or computer system with a large digital storage space that archives all the images. Whereas previously such an archive was a multi-tier archiving system (which meant delays in retrieving old cases), these days with the decreasing cost of digital storage, we have moved towards an always online archiving system. Archives are usually about 10 TB (terabyte) or bigger in size. Refer to Table I for the sizes of imaging examinations.
This is the part of the PACS that is likened to the X-ray storeroom or filing room.

\section{RIS: Radiological Information System ${ }^{6}$}

This is an information system responsible for the management of the workflow (Figs $1 \& 2$ ) and allows the following functions:

- Ordering imaging examinations electronically

- Approving and planning the imaging examinations

- Approving the imaging examinations with the relevant medical fund

- Scheduling the imaging examinations

- Providing the referring doctor and patient with the necessary preparatory information and appointment details for the imaging examination

- Generating a worklist for the radiographers and radiologists

- Distributing and archiving the imaging examination report

- Providing billing and coding information to the HIS (Hospital Information System), practice managers or medical fund provider.

- Providing statistics to the HIS and practice managers.

This is the part of the PACS that is likened to the radiology department administrative division including the approval and scheduling of imaging requests, the establishing of a worklist and ticksheet of examinations performed and the dictations, typing, verification and distribution of radiology reports.

\section{Worklist ${ }^{\top}$}

This is a list of patients and examinations to be performed at an imaging modality or to be reported by a radiologist and is created by the RIS. It is automatically displayed whenever such an imaging modality is turned on or when a radiologist logs on to a station to do their work.

This is the part of the PACS that is likened to the worksheet or ticksheet.

\section{HIS: Hospital Information System ${ }^{8}$}

This is the information system responsible for the electronic health record (EHR). Depending on the system it might only record the demographic data and administrative data. More advanced systems also capture the clinical notes, monitoring equipment, medication and hospital stock used. By definition this is not part of the PACS, but is a crucial part in the functioning of the PACS and RIS.

This part is likened to the medical folder containing all the clinical notes, blood assay results, medication chart and observation chart.

\section{HL7: Health level $7^{8}$}

This is the standard of communication between different information systems managing patient information, specifically the HIS and RIS. the file. 


\section{REVIEW ARTICLE}

\section{IHE: Integrating the Healthcare Enterprise ${ }^{9}$}

This is an initiative by health care professionals and industry to improve the way computer systems in health care share information. It describes how to use standards such as DICOM and HL7 to achieve a specific function.

If you can regard DICOM and HL7 as dictionaries for a specific language, then IHE is a textbook of commonly used phrases.

\section{Legacy system}

This refers to the existing computer system or application program: the antiquated system. Communication with these systems is important, because they contain important data, but the process is normally riddled with compatibility problems.

\section{The digital chain: An integrated equipment-PACS-RIS-HIS solution}

This is the ultimate solution for image management, combining all four systems to improve workflow and productivity. Within the digital working environment, you only achieve the maximal gain once every link in the chain has been digitised.

This part is likened to a department collaboratively working together to achieve common goals.

\section{Conclusion}

We trust that this article will guide you along the path of transition to PACS, and that you will feel less intimidated by the abbreviations used so frequently. In a follow-up article we will identify the current problem areas and expand on the advantages of an integrated PACS-RIS-HIS solution.

1. Choplin RH, Boehme II JM, Maynard CD. PACS mini refresher course: Picture Archiving and Communication Systems: An overview. RadioGraphics 1992; 12: 127-129.

2. Graham RNJ, Perriss RW, Scarsbrook AF. DICOM demystified: A review of digital file formats and their use in radiological practice. Clin Radiol 2005; 60: 1133-1140

3. Stewart BK. PACS mini refresher course: Local Area Network Topologies, Media and Routing. RadioGraphics 1992. 12. 549-566.

4. Bennett WF, Spigos DG, Vaswani KV, Terrell JE. Cable modem access to picture archiving and communication system images using a web browser over the Internet. J Digit Imaging 2000; 13(2 Suppl 1): $93-96$

5. Krupinski EA, Kallergi M. Choosing a radiology workstation: technical and clinical considerations. Radiology 2007; 242: 671-682

6. Thrall JH. Reinventing radiology in the digital age, Part I. The all-digital department. Radiology 2005, 236: 382-385.

7. Gale ME, Gale DR. DICOM modality worklist: an essential component in a PACS environment. J Digit Imaging 2000; 13(3): 101-108.

8. Boochever SS. HIS/RIS/PACS integration: getting to the gold standard. Radiol Manage 2004; 26(3): $16-2$

9. Siegel EL, Channin DS. Integrating the Healthcare Enterprise: A Primer Part 1. Introduction. RadioGraphics 2001; 21: 1339-1341

\section{SONY}

\section{Radiology Imaging for Every Body \& Every Facility}

Sony Digital Radiology Imagers are designed with the practitioner in mind to give users the freedom to pay only for what they need and make maximum savings on capital investment, space and running costs. Able to produce monochrome and colour paper as well as blue diagnostic film hard copies the range can be tailored and matched to specialist applications.

The format size can be varied from 8"x10" up 14"x17" imaging which offers radiologists an even wider range of option at their fingertips. All colour paper images are coated with a special laminate for enhanced durability and reliability. A leader in dry thermal print technology for 25 years, Sony's products offer the benefits of small size, low power use and minimum maintenance.

- Rapid Diagnosis

- Improved Time Management

- On-demand Printing

- Tailored Output

- Can be Installed Vertically or Horizontally

Dealers:

Protea - Greg Walsh: 011-719-5700

Encore Medical - Terry Wiegand: 01 1-777-7500

For the complete range of Medical Solutions by Sony

http://www.sonybiz.net/healthcare or Contact Will Klopper on: 01 1-690-3312

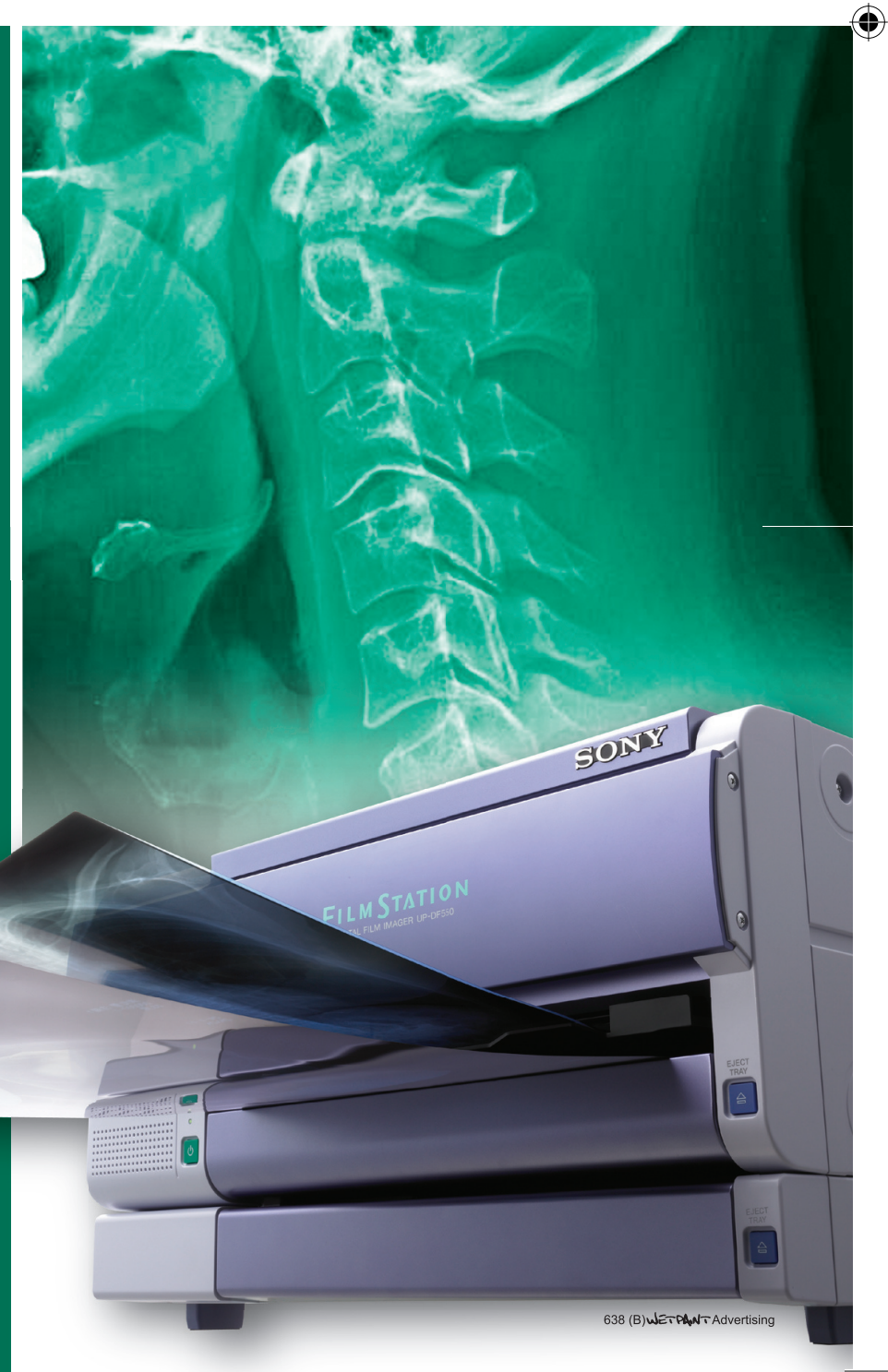

